\title{
Structural evolution in metallomicroemulsions - the effect of increasing alcohol hydrophobicity
}

\author{
Alison Paul*,a, Ian A Fallis*,a, Emily C Stokes ${ }^{\mathrm{a}}$, Stephen M King ${ }^{\mathrm{b}}$ and Peter C Griffiths ${ }^{*, c}$ \\ a School of Chemistry, Cardiff University, Main Building, Park Place, Cardiff CF10 3AT United \\ Kingdom. Fax: +44 (0)2920 874030; Tel: +44 (0)2920 870419; E-mail:paula3@cardiff.ac.uk
}

${ }^{b}$ Science and Technology Facilities Council, ISIS Facility, Rutherford Appleton Laboratory, Didcot, Oxfordshire OX11 OQX

${ }^{c}$ Faculty of Engineering and Science, University of Greenwich, Medway Campus, Central Avenue, Chatham Maritime, ME4 4TB 


\begin{abstract}
Small-angle neutron scattering and contrast variation has been employed to quantify how a series of alcohols with increasing hydrophobicity exert different abilities to structure a model toluene based metallomicroemulsion - a microemulsion system stabilised with a metallosurfactant. Classical microemulsion phase evolution and droplet structure are observed, leading to an oil rich core stabilised by a surfactant film containing a highly concentrated, hydrated metal ion layer.
\end{abstract}

\title{
Introduction
}

Metallosurfactants may be defined as containing a polar transition metal ligand complex in conjunction with a hydrophobic moiety ${ }^{1,2}$, forming a construct in which the physical behaviour of conventional surfactants is combined with the properties of the bound transition metal ion, such as redox ${ }^{3,4,5}$, catalytic ${ }^{6,7}$, photophysical ${ }^{8,9}$ and paramagnetic ${ }^{10,11}$ properties. This has led to their uses in imaging ${ }^{12}$, DNA binding ${ }^{13}$ The classical adsorption and self assembly behaviour of metallosurfactants observed in aqueous solution 14,15,16 affords a high local concentration of the reactive metal species and allows one to constrain a particular characteristic of the metal at an interface in a compartmentalised system, such as a microemulsion.

True metallosurfactants are still a relatively poorly explored class of amphiphile, in particular regarding their ability to form emulsified phases. Microemulsions are an important class of thermodynamically stable, self-assembled structured fluids that find widespread use in a range of applications due to their high internal surface area and the ability to compartmentalise water-insoluble materials in the oil-like region of the microemulsion droplet ${ }^{17}$. Localisation of a reactive metal centre at the air/water interface is an important aspect of catalysis, as demonstrated recently for a decontamination study in which a small proportion of a metallosurfactant was doped into a conventional microemulsion system, increasing the efficiency of reaction with species that partition between the aqueous and oil phases ${ }^{18}$. The ability of metallosurfactants to stablise 
microemulsion phases directly has been demonstrated, and provides the means to further increase the concentration of reactive metal centres at the oil/water interface.

In a previous exploratory study, metallosurfactants comprising copper (II) and zinc (II) as the nitrate complexes of 2,5-dimethyl-7-hydroxy-2,5-diazaheptadecane (scheme 1(a), $\mathbf{L}^{\mathbf{1}}$ ) were found to form stable oil-in-water microemulsions that could solubilise toluene in the presence of butanol ${ }^{19}$. In this paper, the detailed evolution of such microemulsion structures has been investigated to ascertain important formulation parameters, linking composition to structure, stability and partitioning of the various components. To this end solubilisation, phase behaviour and contrast variation small-angle neutron scattering studies have been combined to probe the effect of a homologous series of short chain alcohols as cosolvent/cosurfactants in an aqueous solution of a metallosurfactant. Here, we have used the cyclen based ligand 1-(2-hydroxytetradecyl)-1,4,7,10tetraazacyclononane (scheme $1(\mathrm{~b}), \mathbf{L}^{2}$ ) as the precursor to the copper(II) metallosurfactant $\left[\mathrm{Cu}^{\prime \prime}\left(\mathrm{L}^{2}\right)\right]\left(\mathrm{NO}_{3}\right)_{2}$. We chose this metallosuractant as a means of simplifying the system since this ligand is likely to only form 1:1 metal-ligand adducts, while $\mathbf{L}^{1}$ can potentially form 1:1 and/or 2:1 complexes.

(a)<smiles>CCCCCC(O)CN(C)CCN(C)C</smiles>

(b)

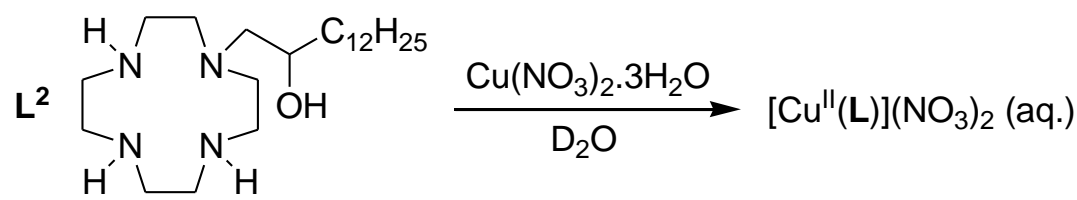

Scheme 1. (a) 2,5-dimethyl-7-hydroxy-2,5-diazaheptadecane $\quad$ (L $\left.{ }^{1}\right) . \quad$ (b) 1-(2hydroxytetradecyl)-1,4,7,10-tetraazacyclononane $\left(\mathrm{L}^{2}\right)$ and the formation the $\mathrm{Cu}(\mathrm{II})$ metallosurfactant $\left[\mathrm{Cu}^{\prime \prime}\left(\mathrm{L}^{2}\right)\right]\left(\mathrm{NO}_{3}\right)_{2}$. 


\section{Experimental}

\section{Synthesis}

Racemic 1-(2-hydroxytetradecyl)-1,4,7,10-tetraazacyclononane (L) was synthesised by the addition of a 10-fold excess of freshly sublimed 1,4,7,10-tetrazacyclododecane to freshly distilled racemic 1,2-epoxytetradecane in ethanol solution by the previously reported method. ${ }^{20}$ The ligand was purified prior to use by bulb-to-bulb distillation on a Büchi kugelrohr apparatus at $0.05 \mathrm{mmHg}$ (oven temp $220^{\circ} \mathrm{C}$ ). A $150 \mathrm{mM}$ standard stock solution of the metallosurfactant was prepared by the addition of an 1.05 equivalents of copper(II)nitrate trihydrate (Sigma Aldrich) to a suspension of the water insoluble $\mathbf{L}$ followed by sonication for 10 minutes to afford a deep blue solution of the metallosurfactant. Phase boundaries were determined at a fixed temperature of $25^{\circ} \mathrm{C}$ by visual inspection. Samples were prepared by sequential addition of toluene and/or alcohol (butanol, octanol or dodecanol) to the above surfactant stock solution.

\section{Small-Angle Neutron Scattering (SANS)}

Small-angle neutron scattering experiments were performed on the fixed-geometry, timeof flight LOQ diffractometer at the ISIS Spallation Neutron Source, Rutherford Appleton Laboratories, Didcot, UK. A Q range between 0.008 and $0.25 \AA^{-1}$ is obtained by using neutron wavelengths $(\lambda)$ spanning 2.2 to $10 \AA$ with a fixed sample-detector of $4.1 \mathrm{~m}$. The samples were contained in $2 \mathrm{~mm}$ path length, UV-spectrophotometer grade, quartz cuvettes (Hellma) and mounted in aluminium holders on top of an enclosed, computercontrolled, sample chamber. Sample volumes were around $0.4 \mathrm{~cm}^{3}$. All experiments were conducted at $30^{\circ} \mathrm{C}$ to avoid close proximity to the phase boundary determined at $25^{\circ} \mathrm{C}$. Temperature control was achieved by using a thermostatic circulating bath pumping fluid through the base of the sample chamber, achieving a temperature stability of $\pm 0.2{ }^{\circ} \mathrm{C}$. Average measuring times were approximately 40 minutes. All scattering data were (a) normalized for the sample transmission, (b) background corrected using a quartz cell filled with $\mathrm{D}_{2} \mathrm{O},(\mathrm{c})$ corrected for the linearity and efficiency of the detector response using the instrument specific software package and (d) put onto an absolute scale by comparison with the scattering from a partially-deuterated polystyrene blend of known molecular weight. 
SANS experiments were performed at selected points across the phase diagram, described relative to $\phi_{\max }$ defined as the visually observable solubility boundary for alcohol and/or toluene $\left(\phi_{\max } R O H\right.$ and $\phi_{\max } T$ respectively). By using combinations of hydrogenous and deuterated oil and/or alcohol, combined with either $\mathrm{H}_{2} \mathrm{O}$ or $\mathrm{D}_{2} \mathrm{O}$ it was possible to produce several scattering "contrasts" at each composition. Each contrast selectively highlights different parts of the system, enabling both the dimensions of individual parts of the droplet and location of specific components to be determined. Fits to the data were carried out using the FISH modelling program ${ }^{21}$, using a core-shell ellipsoid (surfactant+alcohol solutions), or core-shell polydisperse spheres (micromulsions), which are described further in the text. The scattering intensity, $I(Q)$ is given by $V_{p} \phi(\rho 1-$ $\rho 2)^{2} P(Q) S(Q)-B_{\text {inc }}$, where $V_{p}$ is the particle (or micelle) volume, $\phi$ the volume fraction of micelles, $P(Q)$ the form factor which describes micellar size and shape, $S(Q)$ the structure factor that accounts for interactions between micelles. For charged particles (or micelles) the Hayter-Penfold structure factor H-P S(Q) is used, which is described by a charge on the micelle and an effective spherical radius, $S(Q)$ radius, which is indicative of the interaction distance between the micelles. These terms are linked by an inverse Debye screening length accounting for the persistence of charge into solution. Although the $S(Q)$ term is essential to fit the data, previous work ${ }^{22}$ has demonstrated that the fitting process can be insensitive to the individual parameters in the Hayter-Penfold $S(Q)$ structure factor used for micelle-micelle charge repulsions ${ }^{23}$. Hence although in theory it is possible to extract the exact micellar charge (and therefore the degree of counterion dissociation via the aggregation number) from the $S(Q)$ parameters, in the absence of corroborating data the fitted values should be considered as effective, rather than absolute values. Here, such detailed analysis is not possible due to inherent fluorescence quenching by the metallosurfactant which precludes knowledge of the micellar aggregation numbers. 


\section{Results and Discussion}

The ligand $\mathbf{L}^{\mathbf{2}}$ is essentially insoluble in water, and hence the stock metallosurfactant solution was prepared by the addition of a small excess of $\mathrm{Cu}\left(\mathrm{NO}_{3}\right)_{2} \cdot 3 \mathrm{H}_{2} \mathrm{O}$ to a suspension $\mathbf{L}^{\mathbf{2}}$ in $\mathrm{D}_{2} \mathrm{O}$ with sonication, which rapidly afforded a clear deep blue solution. Attempts to crystallise this material failed, affording a deep blue hygroscopic glass on all occasions. The electronic spectrum of this solution was essentially identical to that reported previously for the pentadentate system $\left[\mathrm{Cu}^{\prime \prime}\left(\mathrm{L}^{2}\right)\right](\mathrm{Cl})_{2}$, and thus we tentatively assign the structure of the metallosurfactant to be the analogous pentadentate complex as the nitrate salt $\left[\mathrm{Cu}^{\prime \prime}\left(\mathrm{L}^{2}\right)\right]\left(\mathrm{NO}_{3}\right)_{2}$. The electronic spectrum did not qualitatively change over a wide range of concentrations from $150 \mathrm{mM}$ to $1.5 \mu \mathrm{M}$ indicating essentally no perturbation of the coordination sphere over this concentration range. The high resolution electrospray mass spectrum (1:1 water:MeCN $+0.1 \% \mathrm{HCO}_{2} \mathrm{H}$ carrier, Waters LCT spectrometer) lends confidence this assignment with a double charge molecular ion peak at $\mathrm{m} / \mathrm{z}=225.1670$ (calculated for $\mathrm{C}_{22} \mathrm{H}_{46} \mathrm{DN}_{4} \mathrm{O}^{63} \mathrm{Cu}^{2+}=225.1656$ ), and a singly charged ion peak at $\mathrm{m} / \mathrm{z}=447.3107$ (calculated for $\mathrm{C}_{22} \mathrm{H}_{46} \mathrm{DN}_{4} \mathrm{O}^{63} \mathrm{Cu}^{+}=447.3103$ ), corresponding to a singly deuterium exchanged (amino) ligand as the alcohol complex $\left[{ }^{63} \mathrm{Cu}\left(\mathrm{L}^{2-\mathrm{H}+\mathrm{D})}\right)\right]^{2+}$ and alkoxide complex $\left[{ }^{63} \mathrm{Cu}\left(\mathrm{L}^{2-2 \mathrm{H}+\mathrm{D})}\right)\right]^{+}$. Other ${ }^{63 / 65} \mathrm{Cu}$ and ${ }^{1 / 2} \mathrm{H}$ isotopomers were also observed. The mass spectrum also indicated the absence of free ligand, which is expected given the high formation constants of macroyclic ligand complexes. This latter observation is important as free hydrophobic ligand would significantly perturb the phase behaviour and interpretation of SANS data.

Due to the presence of a metal ion in the headgroup, there is a possibility to use small-angle X-ray scattering (SAXS) to investigate the location of the surfactant headgroups in a micelle, or to identify the surfactant shell around a microemulsion droplet. We have used this approach previously in both types of system, ${ }^{1,19}$ but SAXS measurements cannot provide the level of detail required to determine contributions of different components to microemulsion structure. To do this a multiple contrast small-angle neutron scattering (SANS) experiment is required, using selective deuteration to highlight individual components. The purpose of the SANS experiment is to determine regions of the microemulsion droplet that are occupied by the solvent, surfactant and alcohol. The distance resolution is of the order of several $\mathrm{nm}$, and measurements are long timescale averages of the dynamic structures involved across the 
whole system. In a microemulsion system strong partitioning of the surfactants to the oil/water interface means that all of surfactant is being sampled. Accordingly these measurements are insensitive to small variations in surfactant structure (e.g. trace amounts of hydrocarbon chain homologues), or to the low levels of counterion or surface active impurities that are highly pertinent to surface studies of surfactants (e.g. at the air/water interface), where only a minority of the surfactant is subject to the measurement. ${ }^{24}$

The phase behaviour was determined at $25^{\circ} \mathrm{C}$ by first identifying the solublity limit of the alcohols as binary systems. At characteristic volume fractions of alcohol the samples became cloudy, which we interpret as the solubility limit. A similar approach was adopted with the toluene. For the ternary systems the maximum volume fractions, $\phi_{\max }$, for toluene, butanol, octanol and dodecanol were $0.016,0.06,0.01$ and 0.006 , respectively, as shown in table 1 . With the quarternary systems the phase behaviour was evaluated by sequential addition of toluene into alcohol/surfactant solutions at fixed fractions of the maximum alcohol solubility. The phase boundary was identified as the appearance of droplets of excess toluene were observed to separate out, consistent with the formation of a Winsor I type microemulsion ${ }^{25}$. This phase progression was observed for all alcohols investigated (butanol, hexanol, octanol and dodecanol). As described in table 2, in the absence of alcohols the maximum observed toluene solubility was $\phi=0.016$, whilst in the absence of toluene the maximum solubility of alcohol as a co-surfactant was dependent on the hydrocarbon chain length. At maximum alcohol solubility $\phi_{\max } R O H$, toluene solubility was dependent on alcohol chain length

\section{Ternary systems; addition of alcohol to aqueous surfactant solutions}

SANS data for $150 \mathrm{mM}\left[\mathrm{Cu}^{\prime \prime}\left(\mathrm{L}^{2}\right)\right]\left(\mathrm{NO}_{3}\right)_{2}$ surfactant alone in $\mathrm{D}_{2} \mathrm{O}$ were best fitted to a coreshell ellipsoid model with a Hayter-Penfold structure factor to account for charge-charge repulsions between micelles. The form factor is described by a spherical radius, $R_{\text {micelle }}$ and an ellipticity, $\mathrm{X}$, which is the ratio of non spherical:spherical radii of the ellipse. Hence an $X$ value $>1$ indicates a prolate ellipsoid, and $X<1$ an oblate ellipsoid. The shell thickness, $\delta$, describes a uniform thickness around the core. The fitted parameters, shown in table 
1 , were consistent with previous studies of the same surfactant. The fit and scattering data are shown in figure 1 for comparison purposes to the ternary systems with added alcohols. The characteristic peak in the data arises from spatial seperation of the micelles due to charge-charge repulsion. This is consistent with previous studies, and indicative of degree of surfactant counterion dissociation.
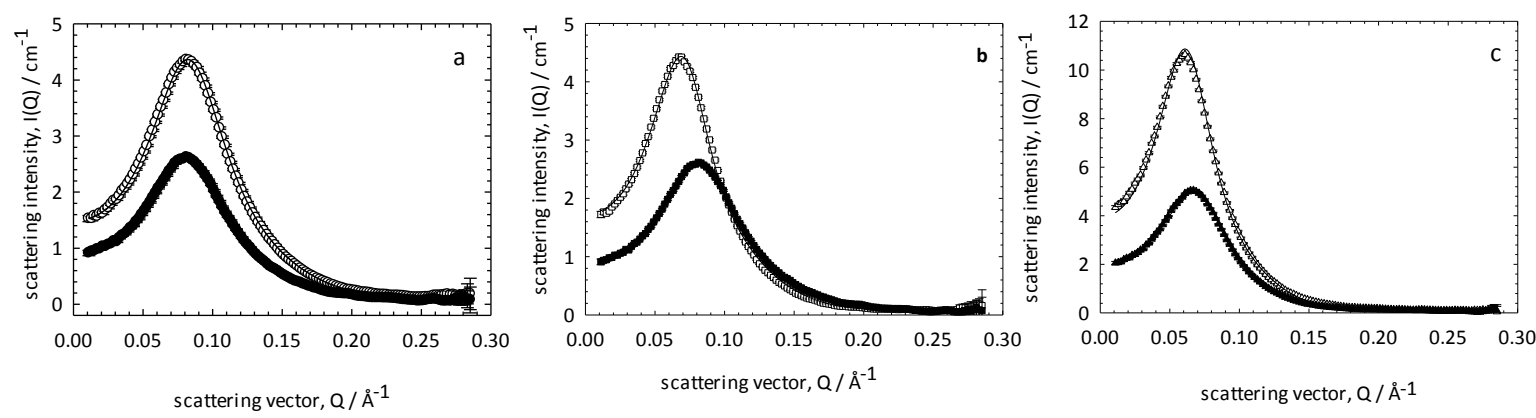

Figure 1: SANS from $150 \mathrm{mM}$ aqueous solutions of $\left[\mathrm{Cu}^{\prime \prime}\left(\mathrm{L}^{2}\right)\right]\left(\mathrm{NO}_{3}\right)_{2}$ with added alcohols at $30^{\circ} \mathrm{C}$. (a) butanol; (b) octanol; (c) dodecanol. Open symbols denote h-alcohols, closed symbols denote $d$-alcohols. Lines are fits to a model for charged ellipsoids, as described in the text.

Our previous study indicated that $\mathrm{Cu}(\mathrm{II})$ metallosurfactants based upon pendant alcohol derivatives of 1,4,7-triazacyclononane (tacn) and 1,4,7,10-tetraazacyclododecane (cyclen) headgroups switched from elliptical to spherical micelles in the presence of butanol, with the alcohol residing mainly in the headgroup region. This was shown using a constrast variation SANS experiment, in which the constrast step changed from the headgroup/water interface for $h$-surfactant/h-butanol in $\mathrm{D}_{2} \mathrm{O}$, to the core/headgroup interface for $h$-surfactant/d-butanol in $\mathrm{D}_{2} \mathrm{O} .{ }^{17}$ With the longer chain surfactant studied here, at these concentrations, no switch in morphology was observed on butanol addition (5 wt\%), with the micelle remaining best fitted by a prolate ellipsoid model. A contrast variation experiment using $h$ - and $d$ - $\mathrm{BuOH}$ provides further information on the alcohol partitioning (figure $1(\mathrm{a})$ ). The scattering curves with $h$ - and $d$-butanol are remarkably similar except for a change in intensity, and this is echoed by the data modelling (parameters given in table 1): with the exception of a change in the scale factor (arising from a the change in constrast term in equation 1 caused by alcohol deuteration), no difference in fitted parameters describing the micelle morphology. 
This absence of a change in the contrast step suggests that unlike the 2,5-dimethyl-7hydroxy-2,5-diazaheptadecane system studied previously $^{1}$ there is no significant partitioning of butanol to the headgroup region. Partitioning of butanol into the core for $\left[\mathrm{Cu}\left(\mathrm{L}^{2}\right)\right]\left(\mathrm{NO}_{3}\right)_{2}$ accounts for the slight change in micelle elongation; butanol solubilsation in the micelle core increases the core volume relative to shell volume, whilst the solublity of butanol in the aqueous phase (cosolvent effect) contributes to screening of charges between headgroups, allowing for closer association and a less curved interface. As described in the experimental, the absolute values for the structure factor parameters cannot be interpreted with the same clarity, but are consistent with this interpretation, indicated by a smaller effective micelle radius and lower micelle charge in the presence of $\varphi_{\max } \mathrm{BuOH}$.

The difference between the two systems $\left[\mathrm{Cu}^{\prime \prime}\left(\mathrm{L}^{2}\right)\right]\left(\mathrm{NO}_{3}\right)_{2}$, and $\left[\mathrm{Cu}^{\prime \prime}\left(\mathrm{L}^{1}\right)\left(\mathrm{H}_{2} \mathrm{O}\right)\right]\left(\mathrm{NO}_{3}\right)_{2}$ likely reflects the more hydrophobic micelle interior afforded by the dodecyl rather than decyl hydrophobic groups, and likely changes in hydration of the headgroup regions in the two micelles. The larger headgroup in the $\left[\mathrm{Cu}^{\prime \prime}\left(\mathrm{L}^{2}\right)\right]\left(\mathrm{NO}_{3}\right)_{2}$ surfactant occupies more of the micelle headgroup region, allowing for less displacement of water by butanol than occurs with $\left[\mathrm{Cu}^{\prime \prime}\left(\mathrm{L}^{1}\right)\left(\mathrm{H}_{2} \mathrm{O}\right)\right]\left(\mathrm{NO}_{3}\right)_{2}$.

Figures 1 (b) and 1 (c) show the same contrast experiment with $h / d$-octanol and $h / d$ dodecanol, respectively. From the fitted parameters when using $h$-alcohols (table 1 ) the micelles for both are more elongated than with butanol ( $X=3$ and 2 , respectively), and the spherical radii are slightly larger $(r=21 \AA \pm 1)$. This elongation of the micelle is consistent with a decrease in average headgroup size leading to a less curved interface. This is supported by the identical fit paramaters obtained for $h$-and $d$-alcohols in both cases (data not shown). Given the very low water solubilities of octanol and dodecanol compared to butanol, this is indicative of the long chain alcohols acting as co-surfactants rather than contributing to a cosolvent effect. 


\begin{tabular}{|c|c|c|c|c|c|c|}
\hline & & & & Fitted paran & eters (SANS) & \\
\hline & & & $R_{\text {micelle }}$ & Ellipticity, $\mathrm{X}$ & $S(Q)$ radius & $S(Q)$ \\
\hline alcohol & $/ w t \%$ & $\phi R O H$ & $/ \AA ̊( \pm 1)$ & $( \pm 0.1)$ & $/ \AA( \pm 2)$ & charge \\
\hline (none) & - & - & 18 & 1.5 & 35 & 25 \\
\hline Butanol & 5 & 0.062 & 17 & 1.6 & 23 & 19 \\
\hline Hexanol & 2 & 0.025 & - & - & - & \\
\hline Octanol & 1 & 0.012 & 21 & 3.0 & 30 & 22 \\
\hline Dodecanol & 0.5 & 0.006 & 22 & 2.0 & 31 & 21 \\
\hline
\end{tabular}

Table 1. Fitted parameters for addition of alcohols at $\phi_{\max }$ to $150 \mathrm{mM}\left[\mathrm{Cu}\left(\mathrm{L}^{2}\right)\right]\left(\mathrm{NO}_{3}\right)_{2}$ at $30^{\circ} \mathrm{C}$.

\section{Ternary systems; addition of toluene to aqueous surfactant solutions}

Figure 2 shows the scattering observed on addition of toluene to the metallosurfactant solution. In panel (a), the use of $d$-toluene and $\mathrm{H}_{2} \mathrm{O}$ allows discrimination between the toluene core and the surfactant shell (core contrast). In panel (c) the scattering from $150 \mathrm{mM}$ metallosurfactant in $\mathrm{D}_{2} \mathrm{O}$ with added $h$-toluene includes the contribution from the surfactant tails (the so-called drop contrast). . Deuterating both the oil and the water highlights the surfactant only (shell contrast), as shown in panel (b). 

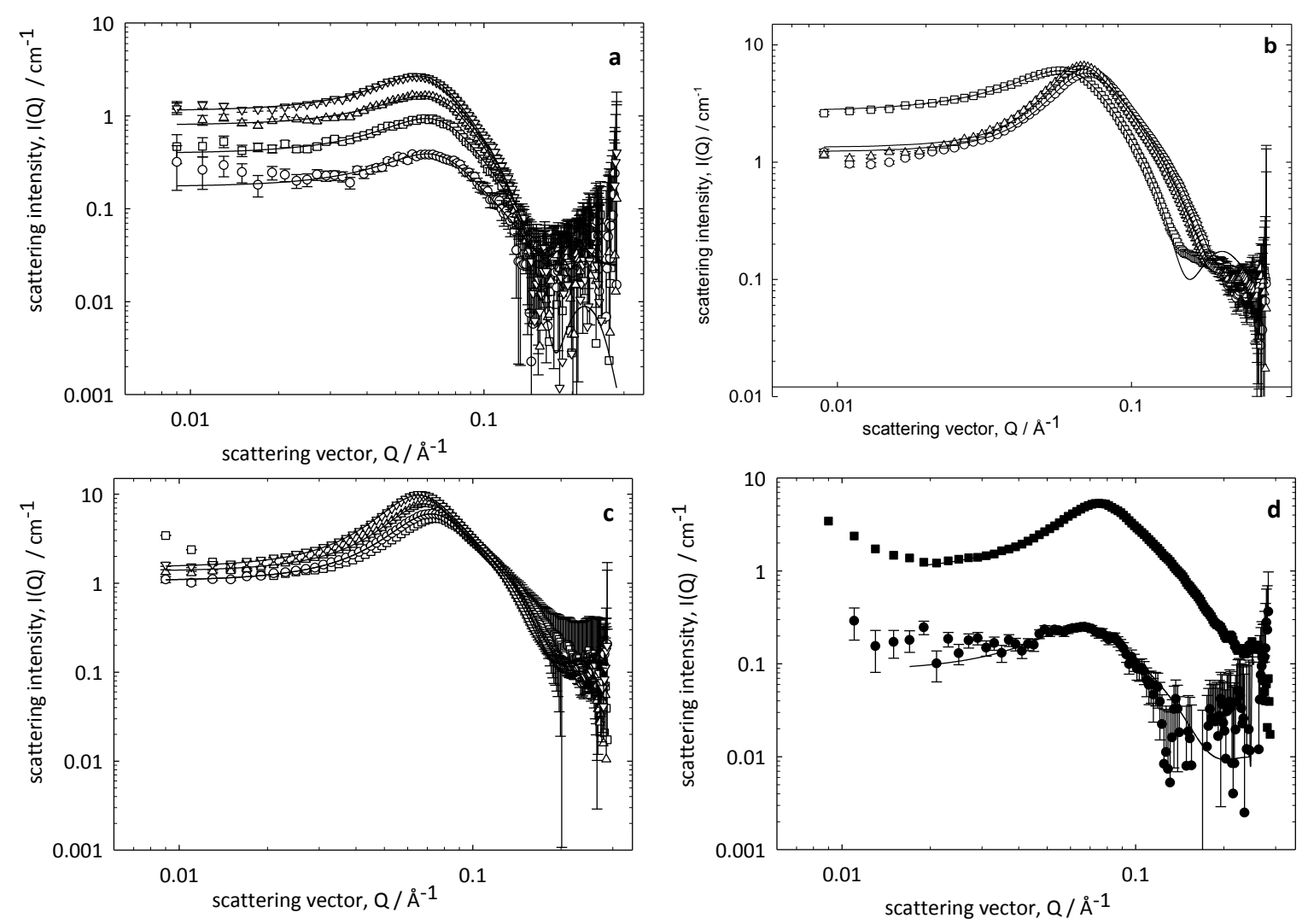

Figure 2. SANS from $150 \mathrm{mM}$ aqueous solutions of $\left[\mathrm{Cu}^{\prime \prime}\left(\mathrm{L}^{2}\right)\right]\left(\mathrm{NO}_{3}\right)_{2}$ with added toluene. (a) d-toluene/h-surfactant/ $\mathrm{H}_{2} \mathrm{O}$ (core contrast); (b) d-toluene/h-surfactant $/ \mathrm{D}_{2} \mathrm{O}$ (shell contrast); (c) h-toluene/h-surfactant/ $\mathrm{D}_{2} \mathrm{O}$ (drop contrast). Symbols denote toluene volume fraction, $\phi_{\text {toluene: }}$ Inverted triangles $=0$; triangles $=0.008$; squares $=0.016$; circles = excess. (d) h-surfactant in $\mathrm{H}_{2} \mathrm{O}$ (circles) and $\mathrm{D}_{2} \mathrm{O}$ (squares), only. Lines are model fits to the data, as described in the text.

The data share several characteristics; noticeable peaks arising arising from interactions between different droplets, and an increase in scattering intensity on addition of toluene associated with changes in droplet structure. Data of this kind are best decribed by a simultaneous fitting of the different contrasts to a theroetical framework that describes the microemulsion structure as a series of concentric, polydisperses spheres - the socalled core-shell model. From this fitting it is possible to extract the core radius and shell thickness of the microemulsion droplet. Changes in these parameters at different contrasts also provide information on partitioning of the oil (and alcohol for quarternary 
systems) between the core, shell and aqueous regions in the system. As very weak scattering is seen for $150 \mathrm{mM}\left[\mathrm{Cu}^{\prime \prime}\left(\mathrm{L}^{2}\right)\right]\left(\mathrm{NO}_{3}\right)_{2}$ in $\mathrm{H}_{2} \mathrm{O}$ (figure $2(\mathrm{~d})$ ) the contrast match is not perfect $\left(I(Q)_{\max }\right.$ is around $0.5 \mathrm{~cm}^{-1}$, compared to $5 \mathrm{~cm}^{-1}$ for $h$-surfactant in $\left.D_{2} O\right)$ so this is accounted for by addition of small contrast step in the data analysis. This complication precluded simultaneous fitting of the three data sets, but the various dimensions obtained from the modelling analysis of each contrast are shown in table 2.

\begin{tabular}{|l|l|l|l|l|c|}
\hline & $\phi_{\text {toluene }}$ & 0 & 0.008 & 0.016 & excess \\
\hline Drop contrast & $R_{\text {drop }}( \pm 1) / \AA$ & 19 & 21 & 28 & 25 \\
& & & & & \\
\hline \multirow{5}{*}{ Core contrast } & $R_{\text {drop }}( \pm 1) / \AA$ & 20 & $24^{a}$ & $27^{a}$ & $30^{a}$ \\
\cline { 2 - 6 } & $R_{\text {core }}( \pm 1) / \AA$ & 20 & 9 & 15 & 19 \\
& $\delta_{\text {shell }}( \pm 1) / \AA$ & & $20^{b}$ & $20^{b}$ & $20^{b}$ \\
\cline { 2 - 6 } & $R_{\text {core }}( \pm 1) / \AA$ & & 24 & 25 & 27 \\
& $\delta_{\text {shell }}( \pm 1) / \AA$ & & $6^{c}$ & $6^{c}$ & $6^{c}$ \\
\hline Shell constrast & $R_{\text {core }}( \pm 1) / \AA$ & 19 & - & 6 & - \\
& $\delta_{\text {shell }}( \pm 1) / \AA$ & 5 & - & 21 & - \\
\hline
\end{tabular}

Table 2. SANS model fit parameters for addition of toluene to $150 \mathrm{mM}\left[\mathrm{Cu}^{\prime \prime}\left(\mathrm{L}^{2}\right)\right]\left(\mathrm{NO}_{3}\right)_{2}$. ${ }^{a}$ single contrast step at water/surfactant headgroups; ${ }^{b}$ shell fixed $(\delta=6 \AA$ ) assuming demixed core and surfactant; ' shell fixed $(\delta=6 \AA$ ) assuming mixed core and surfactant tail.

On addition of toluene to form a microemulsion system, the scattering changed from an elliptical micelle to spherical droplets, as swelling of the micellar core decreases the constrained curvature at the headgroup/water interface. Data were best modelled by polydisperse spheres with a Hayter-Penfold repulsive structure factor. In the absence of toluene fitting to the core-shell contrast model highlighted the interface between the surfactant headgroup and tail. On addition of toluene, ( $d$-toluene/ $h$-surfactant/ $\left.D_{2} O\right)$ the fitted $20 \AA$ shell thickness is consistent with the micelle radii obtained for the surfactant alone in $\mathrm{D}_{2} \mathrm{O}$, around a small $6 \AA$ toluene core. This suggests demixing of the toluene core from the surfactant tail groups in the shell. Due to the incomplete matching of the surfactant in $\mathrm{H}_{2} \mathrm{O}$ (due to the metal headgroup), the core contrast sample ( $d$-toluene/ $h$ surfactant $/ \mathrm{H}_{2} \mathrm{O}$ ) could be satisfactorily fitted to a solid sphere by fixing a single contrast 
step which highlighted the surfactant headgroup $/ \mathrm{H}_{2} \mathrm{O}$ interface. The radii obtained were consistent with those from the drop contrast ( $h$-oil/h-surfactant/ $\left.D_{2} O\right)$. For the true core contrast, fitting the core contrast samples with a fixed shell thickness of $20 \AA$ as per the shell contrast resulted in overestimation of the droplet sizes, and a more satifactory fit was obatined with a $6 \AA$ shell, indicative of the metal headgroups and mixing of the alcohols and surfactant tails with toluene. In both cases the trends are consistent, indicating swelling of the micelles into droplets on addition of toluene.

Quartenary (water, surfactant, alcohol, toluene) systems

Modelling of the changes to the data on addition of alcohols to the toluene containing surfactant solutions yields an insight into the evolution of the microemulsion structure. Taking a point at fixed toluene content, the maximum amount of each alcohol was added to determine the effect on the microemulsion structure. Data are shown at core and drop contrast in figure $3 a$ and $3 b$, with the parameters obtained from model fitting given in table 3. 

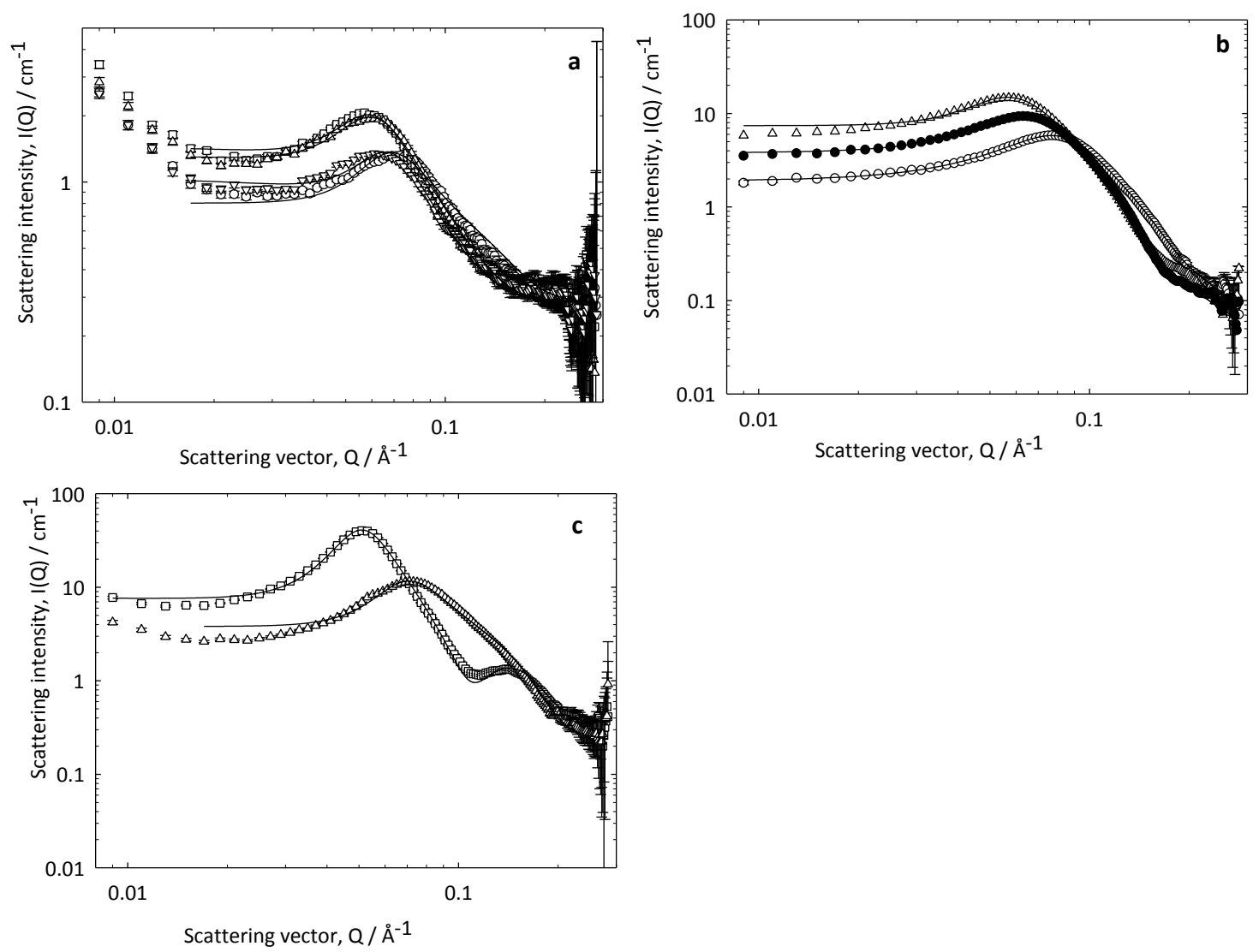

Figure 3. SANS from $150 \mathrm{mM}\left[\mathrm{Cu}^{\prime \prime}\left(\mathrm{L}^{2}\right)\right]\left(\mathrm{NO}_{3}\right)_{2}$ microemulsions with addition of alcohols. (a) core contrast at fixed toluene content ( $\phi_{\text {toluene }}=0.011$ ); (b) drop contrast at fixed toluene content ( $\phi_{\text {toluene }}=0.011$ ); (c) drop contrasts after addition of excess toluene. Alcohol free = filled circles; butanol = open circles; hexanol = inverted triangles; octanol = squares; dodecanol $=$ triangles. Lines are fits to a model for polydisperse spheres as described in the text. Fitted model parameters are given in table 2. 


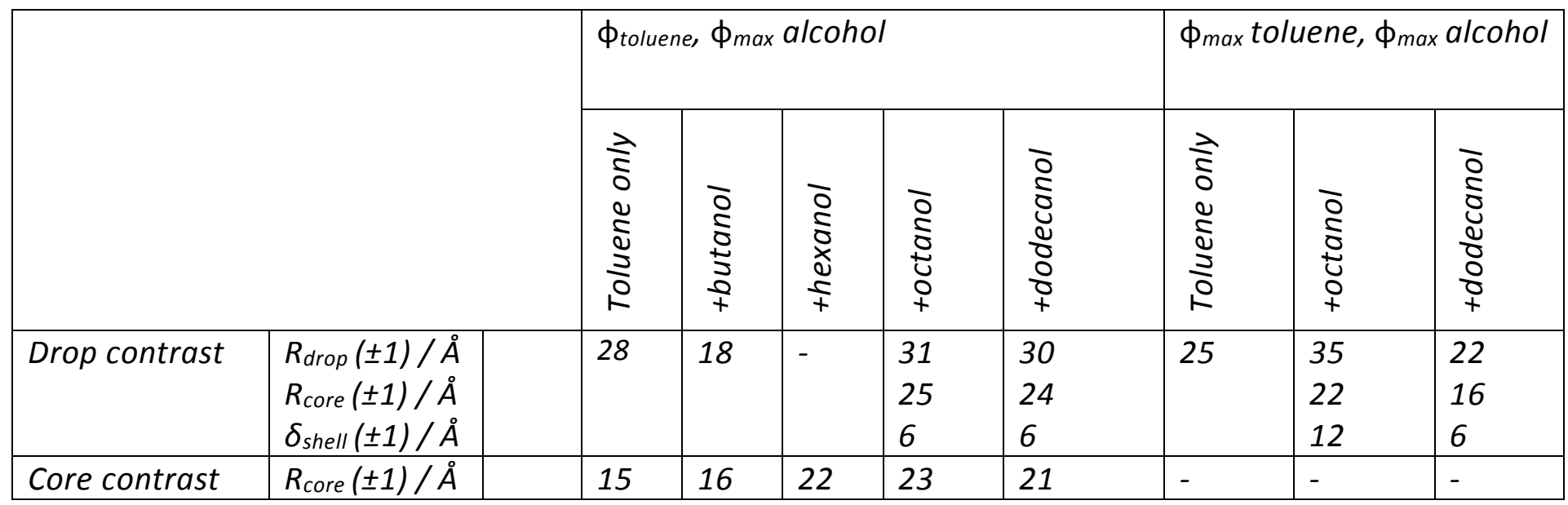

Table 3. Model paramaters from fits to SANS data for $150 \mathrm{mM}\left[\mathrm{Cu}^{\prime \prime}\left(\mathrm{L}^{2}\right)\right]\left(\mathrm{NO}_{3}\right)_{2}$ with added toluene and alcohols.

For butanol, partitioning of the alcohol into the solvent has previously been shown to increase toluene solubility in the continuous phase, thereby decreasing the observed droplet size $\mathrm{e}^{19}$, with contrast variation SANS studies indicating that butanol associated with the droplets remained primarily in the shell region. At drop contrast, a decrease in droplet radius from $28 \AA$ (no alcohol) to $18 \AA$ (with butanol) was observed. However, no change was observed in the core ( $d$-toluene/h-surfactant/h-alcohol/ $\left.\mathrm{H}_{2} \mathrm{O}\right)$ or shell $(d$-toluene $/ h$ surfactant $/ d$-alcohol $/ D_{2} \mathrm{O}$ ) contrasts compared to the alcohol free systems, which rules out the possiblity of butanol distributing throughout the droplet and suggests that the toluene content in the droplets is uniform. These data sets suggest that any butanol associated with the microemulsion droplet simply replaces water in the headgroup region, thereby smoothing out the contrast step between the headgroup region and the solvent, resulting in the smaller observed droplet radius.

The comparable study with hexanol, octanol and dodcecanol led to the formation of larger structures, as shown by the core contrast. This is comensurate with the expected higher degree of partitioning into the droplet due to the changing balance of hydrophobic alkyl chain to hydrophilic (hydroxyl) groups and concommitant decrease in aqueous solubility as chain length increases. Modelling of these systems gave droplet sizes comparable or 
slightly larger (close to the resolution of the experiment) than the alcohol free systems. The best fits to the drop contrast samples was obtained by introducing a contrast step for the surfactant headgroup, as found for the alcohol free systems at higher toluene contents. This is consistent with partitioning of the longer chain alcohols into swollen micellar core.

On addition of excess toluene at maximum alcohol content (Figure $3 c$ ), there is a stiking change in the scattering from the octanol containing sample, which shows "classical" coreshell microemulsion scattering. This is not observed for the dodecanol sample, for which (inline with the alcohol free system) a slight decrease in droplet size is observed. For the octanol system, the change in scattering profiule was fitted by an increased shell thickness of $12 \AA$, indicative of greater toluene content displacing the alcohol into the shell region, where it acts as a cosurfactant to accommodate the decreased curvature associated with the larger droplets.

\section{Conclusions}

Microemulsions are thermodynamically stable systems in which an oil phase is separated from an aqueous continuous phase by a film of surfactant, sometimes in conjunction with a cosurfactant such as an alcohol. Here, we demonstrate how a divalent copper metallosurfactant - a surfactant with a metal ion as an integral part of the covalent headgroup structure - is able to solubilise toluene in the presence of a range of different alcohols. The structure of the microemulsion droplet, the location of the alcohol, and the evolution of the microemulsion structure is elaborated for a series of increasingly hydrophobic alcohols, showing an analogous phase evolution found for traditional surfactants. This will allow classical surfactant behaviour to be used as predictor of metallosurfactants behaviour in microemulsion systems. This detailed understanding will facilitate expansion of the field into areas where more advanced formulation of metallosurfactants is required, providing greater scope to harness the unique range of redox, biomedical and photophysical properties that metallosurfactants possess. 


\section{Conflicts of Interest}

There are no conflicts to declare.

\section{Acknowledgements}

The Science and Technology Facilities Council (STFC) are thanked for facilities access, neutron beamtime and support for consumables. The Life Science Research Network Wales (LSRNW) are thanked sincerely for funding. Abigail Churchill is thanked for preliminary phase studies, and Oliver Burrows for assistance with data reduction and preliminary analysis. Dr Robert Jenkins and Mr Robin Hicks are also gratefully acknowledged for running mass spectra. The School of Chemistry and the College of Physical Sciences and Engineering are gratefully acknowledged for the provision of SAXS facilities.

\section{References}

1 P.C. Griffiths, A. Paul, C. James, G. Brett, R.K. Heenan, I. Grillo, R. Schweins and I.A. Fallis, Soft Matter 2010, 6, 1981-1989

2 P.C. Griffiths, I.A. Fallis, T. Tatchell, L. Bushby and A. Beeby, Adv. Coll. Int. Sci., 2008, 144,13

$3 \quad$ K.S. Wang and G.W. Gokel, J. Phys. Org. Chem.,1997, 10, 323-334;

4 K.S. Wang, S. Munoz, L.T., Zhang, R. Castro, A.E. Kaifer and G.W. Gokel, J. Am. Chem. Soc. 1996, 118, 6707-6715

5 for an early example see F.M. Menger, L.H. Gan, E. Johnson and D.H. Durst, J. Am. Chem. Soc. 1987, 109, 2800-2803

6 S. Bhattacharya and N. Kumari, Coord. Chem. Rev. 2009, 253, 2133-2149

7 F. Mancin, P. Scrimin, P. Tecilla and U. Tonellato, Coord. Chem. Rev. 2009, 253, 21502165

$8 \quad$ P. Wang, S.M. Zakeeruddin, J.E. Moser, M.K. Nazeeruddin, T. Sekiguchi and M. Grätzel, Nature Materials, 2003, 2, 402-407

9 P. Pallavicini, Y.A. Diaz-Fernandez and L. Pasotti, Coord. Chem. Rev. 2009, 253, 22262240

10 R. Hövland, C. Glogard, A.J. Aasen and J. Klaveness, J. Chem Soc. Perkin Trans 2, 2001, 929-933 
11 A. Accardo, D. Tesauro, L. Aloj, C. Pedone, and G. Morelli, Coord. Chem. Rev. 2009, 253, 2193-2213

12 Q. Zhu, F. Pan, U. Tian, A. Hu, 2016, RSC Advances, 6, 29441-29447

13 V. Krishnaveni, N. Kumaraguru, J. Pharm. Med. Res., 2017, 3, 68-72

14 R. Humphry-Baker, Y. Moroi, M. Grätzel, E. Pelizzetti and P. Tundo, J. Am. Chem. Soc, 980, 102, 3698

15 Y. Moroi, A.M. Braun, M. Grätzel, J.Am.Chem.Soc., 1979, 101, 567

16 S.A. Alkaitis, G. Beck and M. Grätzel, J. Am. Chem. Soc., 1975, 97, 5723

17 A. Yaghmur and O. Glatter, Adv. Coll. Int. Sci., 2009, 147, 333

18 I.A. Fallis, T. Cosgrove, C.A. Dreiss, N. Govan, M. Hargreaves, R.K. Heenan, I. Holden, S.J. Mitchell, J.A. Platts, G.M. Williams and P.C. Griffiths, J. Am. Chem. Soc., 2009, 131, 9746

19 A. Paul, I. Fallis, C. Cooper, T. Wess, K. Thomas, R.K. Heenan, S.M. King and P.C. Griffiths Soft Matter 2010, 6, 2552-2556

20 P. C. Griffiths, I. A. Fallis, D. J. Willock, A. Paul, C. L. Barrie, P. M. Griffiths, G. M. Williams, S. M. King, R. K. Heenan, and R. Görgl, Chem.Eur J. 2004, 10, $2022-2028$

21 FISH Data Analysis Software, R.K. Heenan, ISIS STFC Facility, Personal Communication

22 A. Paul, P C Griffiths, E Pettersson, P Stilbs, B Bales, R Zana, R J Heenan, J. Phys. Chem. B, 2005, 109, 15775-15779

23 J. B. Hayter, J. Penfold, J. Mol. Phys. 1981, 42, 109-118.

24 J. Eastoe, S. Nave, A. Downer, A. Paul, A. Rankin, K. Tribe and J. Penfold, Langmuir 2000, 16, 4511-4518

25

P. Winsor P, Trans. Faraday Soc., 1948, 44, 376 